\title{
Stage IV Renal Pelvis and Ureter Cancer AJCC v8
}

National Cancer Institute

\section{Source}

National Cancer Institute. Stage IV Renal Pelvis and Ureter Cancer A/CC v8. NCI

Thesaurus. Code C140373.

Stage IV includes: (T4, N0, M0); (Any T, N1, M0); (Any T, N2, M0); (Any T, Any N, M1). T4: Tumor invades adjacent organs, or through the kidney into the perinephric fat. N0: No regional lymph node metastasis. N1: Metastasis in a single lymph node, $2 \mathrm{~cm}$ or less in greatest dimension. N2: Metastasis in a single lymph node, more than $2 \mathrm{~cm}$ in greatest dimension; or multiple lymph nodes. M0: No distant metastasis. M1: Distant metastasis. (AJCC 8th ed.) 